\title{
Scaling and renormalization in the modern theory of polarization: Application to disordered systems
}

\author{
Balázs Hetényi $\odot,{ }^{1,2,3}$ Selçuk Parlak $\odot,{ }^{3}$ and Mohammad Yahyavi $\oplus^{3}$ \\ ${ }^{1}$ Department of Theoretical Physics, Institute of Physics, Budapest University of Technology and Economics, \\ Budafoki út 8, H-1111, Budapest, Hungary \\ ${ }^{2}$ MTA-BME Topology and Correlations Research Group, Department of Theoretical Physics, \\ Budapest University of Technology and Economics, H-1521 Budapest, Hungary \\ ${ }^{3}$ Department of Physics, Bilkent University, TR-06800 Bilkent, Ankara, Turkey
}

(Received 28 August 2021; revised 18 October 2021; accepted 3 December 2021; published 15 December 2021)

\begin{abstract}
We develop a scaling theory and a renormalization technique in the context of the modern theory of polarization. The central idea is to use the characteristic function (also known as the polarization amplitude) in place of the free energy in the scaling theory and in place of the Boltzmann probability in a position-space renormalization scheme. We derive a scaling relation between critical exponents which we test in a variety of models in one and two dimensions. We then apply the renormalization to disordered systems. In one dimension, the renormalized disorder strength tends to infinity, indicating the entire absence of extended states. Zero (infinite) disorder is a repulsive (attractive) fixed point. In two and three dimensions, at small system sizes, two additional fixed points appear, both at finite disorder: $W_{a}\left(W_{r}\right)$ is attractive (repulsive) such that $W_{a}<W_{r}$. In three dimensions, $W_{a}$ tends to zero and $W_{r}$ remains finite, indicating a metal-insulator transition at finite disorder. In two dimensions, we are limited by system size, but we find that both $W_{a}$ and $W_{r}$ decrease significantly as system size is increased.
\end{abstract}

DOI: 10.1103/PhysRevB.104.214207

\section{INTRODUCTION}

Scaling theory of localization in disordered systems [1-4] has a long history. A milestone work by Abrahams et al. [1], often referred to as the "gang of four" paper (G4), put forth this theory to explain the dimensional dependence of criticality. The central results for systems of no symmetry are that all states are localized in one dimension (1D) and even in two dimensions (2D) there are no extended states, but here a crossover occurs and only in three dimensions (3D) does a true metal-insulator transition occur in the form of an unstable fixed point.

Experimental evidence shows unambiguous support for the G4 conclusions in 1D [5,6] and 3D [7-9]. The 2D results were more difficult to establish [10] experimentally due to the possibility of weak localization [11-13]. Theoretically, a debate [14-16] about a possible metal-insulator transition in 2D, rather than the entire absence of extended states, arose.

An important development in the understanding of crystalline systems in general (with or without disorder) was the development of the modern theory of polarization [17-19] (MTP). This theory provides the tools $[20,21]$ to measure localization. Although some studies [22-24] have used these tools to assess localization in disordered systems, how the G4 results concur with the MTP is still an open question.

In this paper, we seek to fill this gap by developing thermodynamic scaling and renormalization methods [25-27] within the MTP context. The idea is to use the MTP characteristic function (also known as the polarization amplitude [28]) in place of the partition function as a starting point for both scaling (similar to Widom scaling of critical exponents) and our position-space renormalization scheme.
In 1D, our flow lines tend to a high disorder attractive fixed point, meaning that there are no extended states. In 2D and $3 \mathrm{D}$, for finite system sizes, we find an attractive $\left(W_{a}\right)$ and a repulsive $\left(W_{r}\right)$ fixed point $\left(W_{a}<W_{r}\right)$. The key question becomes how these fixed points evolve with system size. In $3 \mathrm{D}, W_{a}$ tends to zero, while $W_{r}$ tends to a finite number, indicating a metal-insulator transition. In 2D, both the $W_{a}$ and $W_{r}$ decrease, but due to finite size limitations, it is difficult to draw a definite conclusion that is valid for the thermodynamic limit. We discuss the possible scenarios.

Other renormalization approaches to Anderson localized systems, apart from the G4 scaling theory, have also been used [29-32]. Traditional real-space renormalization schemes, based on blocking sites of the lattice (MigdalKadanoff procedure), concur with G4. In the context of MTP, renormalization has been applied by Voit and Nakamura [33], but this technique relies on bosonization and is only applicable in $1 \mathrm{D}$.

There are many investigations [28,34-38] which focus on the distribution function and the scaling of the polarization amplitude in band theoretic and correlated quantum models. MTP also serves as the starting point for deriving topological invariants [39], for example, the time-reversal polarization; the topological invariant in the Fu-Kane spin pump [40] is the difference of the Zak phases of different members of a Kramers pair.

In Sec. II, the scaling theory of localization and the MTP are outlined and the motivation for this work is stated, also placing our work in a contemporary context. In Sec. III, the Hamiltonians of the models studied in this work are given. In Sec. IV, we derive a relation between critical exponents based on Widom's thermodynamic scaling and test it for some 
model systems. In Sec. V, we develop our renormalization approach and apply it to disordered systems in different dimensions. In Sec. VI, we conclude our work.

\section{BACKGROUND AND MOTIVATION}

The starting point of the G4 scaling theory [1] is the specification of the Thouless number [41] as the relevant quantity to analyze. The Thouless number is a dimensionless conductance defined as

$$
g(L)=\frac{G}{e^{2} / 2 \hbar},
$$

where

$$
G=\frac{\Delta E}{d E / d N}
$$

where $\Delta E$ is the difference between energy levels calculated using periodic boundary conditions and antiperiodic boundary conditions, and $d E / d N$ is the average level spacing. The argument in support of $G$ as a conductance is that it is localization that determines whether or not a state is insulating. A delocalized state should be sensitive to changing the boundary conditions, whereas a localized one should not. G4 then argues that the $g(L)$ depends only on the system size. The scaling theory then analyzes the scaling function,

$$
\beta(g)=\frac{d \ln g}{d \ln L} .
$$

In this function, $L$ appears explicitly, since in disordered systems the system size dependence is more pronounced than in clean systems. Asymptotic analysis can be applied. When the conductance is small (states are localized), it is expected that

$$
g=\exp (-L / \xi)
$$

where $\xi$ denotes a correlation length. When extended states dominate, the conductance is expected to behave as

$$
g=\sigma_{0} L^{d-2},
$$

where $\sigma_{0}$ denotes the conductivity and $d$ is the dimensionality of the system. From this information, by plotting $\beta(g)$ as a function of $\ln g$, the dimensional dependence of the critical behavior can be surmised. In 1D, all states are localized since the function $\beta(g)$ is always negative, even as $\ln g$ goes to infinity. In 2D, the curve is negative when $\ln g$ is negative, and it approaches zero as $\ln g$ goes to infinity, meaning that even in 2D, the states are extended; however, in this case, due to the crossover between exponential and logarithmic behavior, G4 predicts that experiments may detect a sharp mobility edge. In $3 \mathrm{D}$, since the curve crosses $\beta(g)=0$, corresponding to an unstable fixed point, a metal-insulator transition is predicted.

Overall, in assessing a metal-insulator transition in (ordered or disordered) many-body quantum systems, the 1964 work of Kohn [42] was a starting point. On the one hand, Kohn argued [42] that assessing whether a system is metallic or insulating can be done by investigating the response of the system to twisting the boundary conditions. On the other hand, Kohn also pointed out that the localization of the center of mass of the charge distribution is the ultimate measure of whether a system is conducting or insulating. The two approaches are equivalent.
The central difficulty addressed by MTP was the ill-defined nature of the position operator in systems with periodic boundary conditions. This difficulty hindered the application of the hypothesis of Kohn [42] in calculations for crystalline systems. The problem was overcome by casting the polarization in terms of a geometric phase [43] of the Zak [44] variety, which arises upon integrating across the Brillouin zone. Other relevant properties, such as the many-body generalization [20] of the polarization and the variance thereof [21], were also derived. The program of Kohn [42] was later realized through the MTP [17-19,21].

In the formalism of Resta and Sorella, the variance of the total position is cast [21] as

$$
\chi^{(2)}=\sigma^{2}=-\frac{L^{2}}{2 \pi^{2}} \operatorname{Re} \ln Z_{1},
$$

where

$$
Z_{q}=\left\langle\Psi_{0}\left|\exp \left(i \frac{2 \pi q}{L} \hat{X}\right)\right| \Psi_{0}\right\rangle,
$$

and where $\left|\Psi_{0}\right\rangle$ denotes a quantum ground state, $q$ is an integer, and the total position operator is defined as

$$
\hat{X}=\sum_{j=1}^{L} \hat{n}_{j} j
$$

where $\hat{n}_{j}$ is the density operator at site $j$. If $\sigma$ tends to infinity with system size, the system is metallic.

In this work, we perform calculations for disordered systems in different dimensions based on MTP. Since the Thouless number is a measure of localization, we replace it with the quantity from the MTP which can be taken as its analog,

$$
g(L) \rightarrow 1-\left|Z_{1}\right|
$$

This is not an exact correspondence by any means. We justify it by first stating that the variance can be cast according to an approximation [38] different from the one of Resta and Sorella,

$$
\sigma^{2}=\frac{L^{2}}{2 \pi^{2}}\left(1-\left|Z_{1}\right|\right) .
$$

This approximation has the advantage that in the limit of the Fermi sea $\left(Z_{1}=0\right), \sigma$ scales with system size linearly, which is expected on robust physical grounds. Furthermore, in the inset of Fig. 2(a), we show the quantity $1-\left|Z_{1}\right|$ as a function of the Thouless number (defined as the sum over the absolute value of the difference in energy between periodic and antiperiodic boundary conditions, divided by the total energy difference), for a 1D system of $L=160$ and averaged over 100 disorder realizations (replicas) [Hamiltonian in Eq. (11)]. The function is monotonic, which also justifies our replacing of the Thouless number with $\left|Z_{1}\right|$ in our renormalization scheme (Sec. V).

To perform the asymptotic analysis, the G4 scaling theory uses two pieces of information. In the small conductivity (large disorder) limit, it is assumed that the conductivity localizes exponentially [Eq. (4)]. In the opposite limit, the conductance is related to the conductivity via the relation given by Eq. (5). In the latter, there appears to be no direct 
prescription to calculate the conductance based on a microscopic Hamiltonian. To phrase the question differently: given a disordered Hamiltonian for which we can calculate the eigenstates, how do we calculate the conductance? Which states do we consider? Should we consider a distribution of states? In our calculations below, we will average the relevant quantities over all states. This amounts to a high-temperature approximation since all states have the same contribution. The position operator that we use [45-47] is a single-body one.

\section{MODELS}

Most of this paper is devoted to disordered systems. The one-dimensional version of the disordered Hamiltonian that we study can be written as

$$
\hat{H}=\sum_{i=1}^{L}\left[-t\left(\hat{c}_{i}^{\dagger} \hat{c}_{i+1}+\text { H.c. }\right)+W \xi_{i} \hat{n}_{i}\right],
$$

where $t$ denotes the hopping parameter (which will be taken as the unit of energy), $W$ indicates the disorder strength, and $\xi_{i}$ is a normal distributed random number. On the other hand, in the next section we also test the result of our Widom scaling theory for the Su-Schrieffer-Heeger [48] (SSH) and Rice-Mele [49] (RM) models. The latter can be written as

$$
\hat{H}=\sum_{i=1}^{L}\left[-\left(t+(-1)^{i} \delta t\right)\left(c_{i}^{\dagger} c_{i+1}+\text { H.c. }\right)+\Delta(-1)^{i} \hat{n}_{i}\right] \text {, }
$$

where $\delta t$ denotes the alternation in hopping and $\Delta$ denotes the strength of an alternating on-site potential. The SSH Hamiltonian is obtained by setting $\Delta=0$ in Eq. (12).

\section{WIDOM SCALING IN THE MODERN THEORY OF POLARIZATION}

We consider an $N$-electron system that is one dimensional for convenience and periodic in $L$. The discrete analog of the characteristic function is given in Eqs. (7) and (8). The expression for the average position in a many-body crystalline system given by Resta is

$$
\langle X\rangle=\frac{L}{2 \pi} \operatorname{Im} \ln Z_{1},
$$

which reduces to the Zak phase if $\Psi_{0}$ is a Slater determinant. The average position [20] [Eq. (13)] and the variance [21] [Eq. (6)] are finite difference derivatives of $\ln Z_{q}$ at $q=0$, i.e., first and second derivatives, respectively.

For our purposes in this section, it is more suitable to take the thermodynamic limit in Eq. (7) and write

$$
Z(K)=\left\langle\Psi_{0}|\exp (i K \hat{X})| \Psi_{0}\right\rangle,
$$

and express the variance as

$$
\chi^{(2)}=-\left.\frac{\partial^{2} \ln Z(K)}{\partial K^{2}}\right|_{K=0} .
$$

The quantity $\chi^{(2)}$ can be interpreted as a semiclassical approximation to the dielectric susceptibility. It is to be expected that if a phase transition point is approached from the insulating side, $\chi^{(2)}$ diverges.

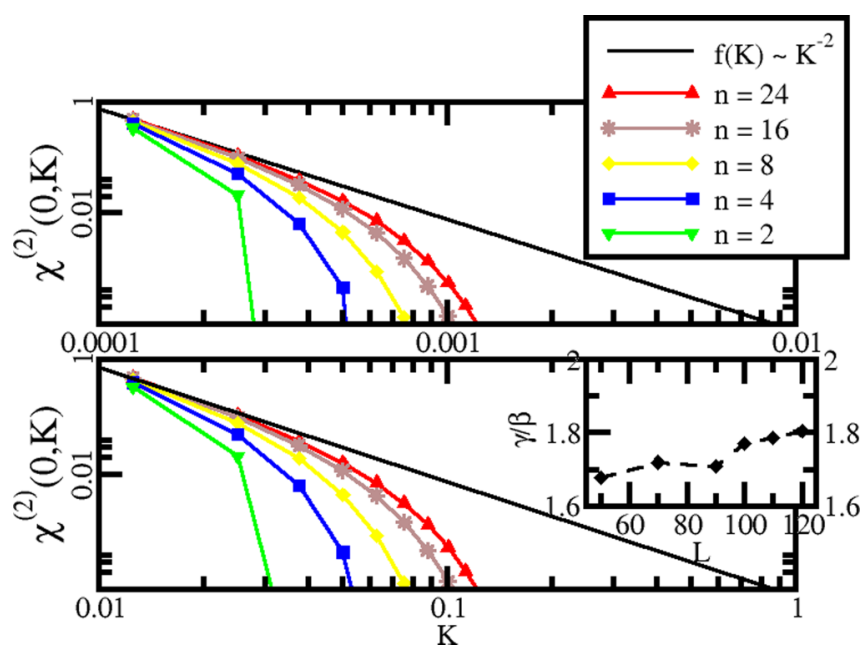

FIG. 1. Log-log plot of $\chi^{(2)}(0, K)$ as a function of $K$ for different order, finite difference approximations to the second derivative [see Eq. (16)] for two system sizes. $n$ refers to the order of the approximation, $O\left(K^{-2 n}\right)$. The upper panel shows results for $L=100000$ and the lower panel for $L=1000$. The solid line indicates a curve of the form $f(K) \propto K^{-2}$. The legend is for the upper and lower panels, not for the inset of the lower panel. The inset of the lower panel shows the estimated ratio of the exponents $\gamma$ and $\beta$ as a function of system size for a two-dimensional disordered system.

To keep the discussion general, we introduce a variable $D$ which characterizes the approach to the critical point (we assume that $D=0$ is a quantum phase transition or gap closure point). We first define

$$
\chi^{(n)}(D, K)=\frac{1}{i^{n}} \frac{\partial^{n} \ln Z(D, K)}{\partial K^{n}},
$$

and also define three critical exponents characterizing the approach to the phase transition:

$$
\begin{aligned}
& \chi^{(2)}(D, 0) \propto \frac{1}{D^{\beta}}, \\
& \chi^{(4)}(D, 0) \propto \frac{1}{D^{\gamma}}, \\
& \chi^{(2)}(0, K) \propto \frac{1}{K^{\delta}} .
\end{aligned}
$$

We also define the singular quantity

$$
\Phi(D, K)=-\ln Z(D, K)
$$

which serves as the analog of the free energy. Applying the scaling relation $\Phi\left(\lambda^{a} D, \lambda^{b} K\right)=\lambda \Phi(D, K)$, we obtain the following relation between the scaling exponents:

$$
\gamma \delta=\beta(\delta+2) .
$$

We tested this relation on a number of different models. For the exponent $\delta$ ( $D=0$ limit corresponds to the Fermi sea), our calculations are shown in Fig. 1. The different panels show different system sizes; the different curves within each panel are finite difference approximations to the second derivative [Eq. (17)]. From these calculations, we can conclude that $\delta=$ 2 , which also means that $\gamma=2 \beta$. We then calculated the other exponents of the SSH and Rice-Mele [Eq. (12)] models, in 1D and $2 \mathrm{D}$, and found that $\beta=2$ and $\gamma=4$. 
In addition, we also calculated the exponents for a $1 \mathrm{D}$ disordered system with the Hamiltonian given by Eq. (11). When calculating the cumulants [Eq. (17)], a difficulty that was faced was that a smooth curve only results for large systems. In particular, at small $W$, large system sizes are needed for converged results. We calculated the critical exponents for an $L=1280$ system, averaged over 100 realizations of the disorder, and found $\beta=3.61(5)$ and $\gamma=7.16(27)$, consistent with Eq. (19). We also made calculations for three different realizations of the disorder (three different calculations, where no disorder average is taken and only $W$ is swept) for a system of size $L=2560$. The results for the exponents turned out to be $\beta=3.53(4), 3.45(4), 3.52(5)$ and $\gamma=7.12(9), 6.90(12), 7.07(11)$, respectively, again consistent with Eq. (19) within error bars.

We also performed a calculation for a $2 \mathrm{D}$ disordered system. In this calculation, we encountered system size related difficulties; however, we provide an estimate of the ratios of critical exponents (inset of the lower panel of Fig. 1). As shown below, at the accessible system sizes, there is a transition in two-dimensional disordered systems (see Fig. 2), but the position of this transition decreases as the system size is increased (see Fig. 4, upper panel). The relevant range of $\chi^{(2)}$ and $\chi^{(4)}$ as a function of $W$ is above the transition (which itself changes with system size), but before the disorder becomes too large because then the errors are larger than the values of $\chi^{(2)}$ and $\chi^{(4)}$. We estimated the critical exponents $\gamma$ and $\beta$ by looking for the maxima of the derivatives of the functions $\log \chi^{(2)}$ and $\log \chi^{(4)}$ as a function of the logarithm of disorder strength. This estimate is shown in the inset of the lower panel of Fig. 1. The estimated ratio is increasing for the range of system sizes studied, and it is $\gamma / \beta \approx 1.8$ for the largest size, $L=120$.

\section{REAL-SPACE RENORMALIZATION IN THE MODERN THEORY OF POLARIZATION}

In real-space renormalization [25-27], one starts with the blocking of sites of the lattice. To each block, a single block variable is assigned. The blocked system is assumed to have the same Hamiltonian as the original Hamiltonian (although this can mean an extended set of couplings). The parameters of the blocked Hamiltonian are tuned to produce the same Boltzmann probability as the original Hamiltonian, provided that the configurations of the starting Hamiltonian consistent with a given configuration of the block variables are summed over (traced out).

A common way to define a blocked system is by the procedure of "decimation," in which some of the variables of the original system are traced out. In most cases, the set of equations obtained this way is overdetermined, so either new parameters have to be introduced (for example, by extending the couplings included in the Hamiltonian) or further approximations have to be introduced, for example equating cumulants [50], between the original and the renormalized system, rather than the full Boltzmann distribution.

We now apply this set of steps to the quantity

$$
\tilde{P}_{j}\left(x_{1}, \ldots, x_{L}\right)=\left|\Psi_{0}\left(x_{1}, \ldots, x_{L}\right)\right|^{2} \exp \left(i \frac{2 \pi}{L} \hat{X}\right) .
$$
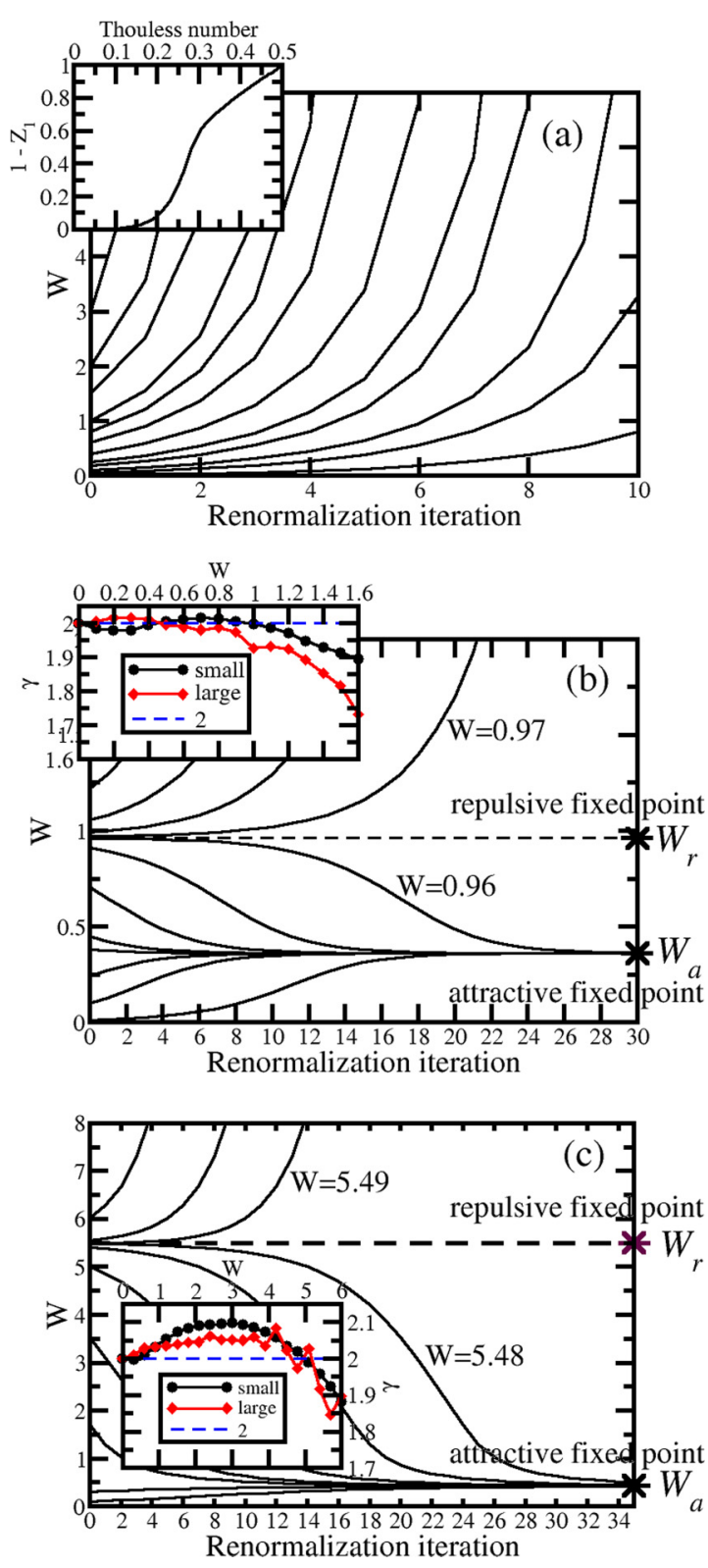

FIG. 2. Renormalization flow lines for disordered systems of different dimensions. (a) One-dimensional $L=160,100$ replicas averaged. The inset shows the quantity $1-Z_{1}$ vs the Thouless number. (b) Two-dimensional, $L=24,100$ replicas averaged. The repulsive $\left(W_{r}\right)$ and attractive $\left(W_{a}\right)$ fixed points are also indicated. The inset shows the size scaling exponent calculated using system sizes of $L=12,14,16,18$ (small) and $L=72,80,96$ (large). (c) Threedimensional, $L=8,100$ replicas averaged. The repulsive $\left(W_{r}\right)$ and attractive $\left(W_{a}\right)$ fixed points are also indicated. The inset shows the size scaling exponent calculated using system sizes of $L=6,8,10$ (small) and $L=20,22,24$ (large).

Here, the index $j$ indicates the step in the renormalization. As a first step, we integrate out all the odd sites, resulting in

$$
\tilde{P}_{j+1}\left(x_{2}, \ldots, x_{L}\right)=\iint d x_{1} \ldots d x_{L-1} \tilde{P}_{j}\left(x_{1}, \ldots, x_{L}\right) .
$$

We now require that the $\tilde{P}_{j}\left(x_{2}, \ldots, x_{L}\right)$ of the remaining variables equals $\tilde{P}_{j+1}\left(x_{2}, \ldots, x_{L}\right)$. It is easy to see, based on the definition of $\hat{X}$ [Eq. (8)], that $\tilde{P}_{j}\left(x_{2}, \ldots, x_{L}\right)$ corresponds to 

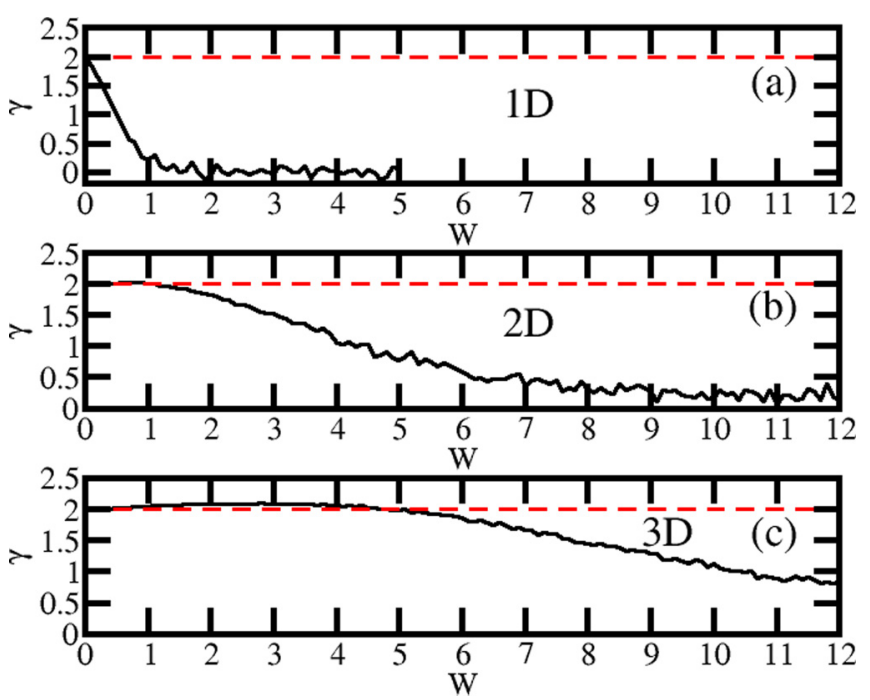

FIG. 3. Size scaling exponent of the variance of the polarization for a (a) one-dimensional, (b) two-dimensional, and (c) threedimensional system.

the distribution of a system with half the size of the original one, $L / 2$. As in other real-space renormalization techniques, the requirement is too stringent, so to arrive at a practical scheme, we use the relaxed requirement,

$$
Z_{1}\left(W_{j+1}, L / 2\right)=Z_{1}\left(W_{j}, L\right) .
$$

In other words, in each renormalization step, with a given disorder strength and system size, we find the disorder strength at half the system size which generates the same value of $Z_{1}\left(W_{j}, L\right)$. It is not the entire probability distribution that is kept fixed in the course of a renormalization step, but only one Fourier mode of the distribution of the many-body position. In this sense, this renormalization is tailored to MTP.

In Fig. 2, the flow lines of the renormalization scheme are shown for systems of different dimensions. Figure 3 shows the size scaling exponent $(\gamma)$ of the variance of the polarization. We define $\gamma$ as

$$
\chi^{(2)}=a L^{\gamma} .
$$

In previous studies [38], it was shown that metal-insulator transitions can be accurately determined by investigating $\gamma$. In clean systems, a gapless system will exhibit $\gamma=2$, while in gapped insulators, $\gamma \rightarrow 1$.

Figure 2(a) shows results for a 1D disordered system of size $L=160$, and 100 realizations of the disorder averaged. All flow lines which start at a finite value tend towards infinity, indicating that all states are localized. We found no significant size dependence or dependence on the number of replicas. Also, Fig. 3(a) is consistent with the renormalization flows. The clean conducting system $(W=0)$ exhibits $\gamma=2$, and finite disorder strength leads to a rapid decrease in $\gamma$. These results concur with the G4 [1].

Figure 2(b) shows the flow lines in a 2D sample calculation. The linear dimension of the system is $L=24$, and the calculation was done on a square lattice. The number of disorder configurations averaged was 100 . The flow lines here show qualitatively different behavior from the $1 \mathrm{D}$ case. We find two fixed points on the $W$ axis, with one repulsive, $W_{r} \approx 1$,
TABLE I. The repulsive $\left(W_{r}\right)$ and attractive $\left(W_{a}\right)$ fixed points for three sample calculations as a function of system size for a twodimensional disordered system. The number of replicas is indicated in parentheses.

\begin{tabular}{lcccccc}
\hline \hline Size (No. replicas) & $W_{r}(1)$ & $W_{r}(2)$ & $W_{r}(3)$ & $W_{a}(1)$ & $W_{a}(2)$ & $W_{a}(3)$ \\
\hline $24 \times 24(100)$ & 1.00 & 0.86 & 0.95 & 0.38 & 0.40 & 0.43 \\
$32 \times 32(50)$ & 0.78 & 0.85 & 0.84 & 0.30 & 0.29 & 0.29 \\
$48 \times 48(25)$ & 0.69 & 0.75 & 0.69 & 0.19 & 0.17 & 0.16 \\
$64 \times 64(15)$ & 0.65 & 0.66 & 0.65 & 0.15 & 0.14 & 0.14 \\
$80 \times 80(10)$ & 0.60 & 0.62 & 0.62 & 0.11 & 0.10 & 0.12 \\
$96 \times 96(5)$ & 0.57 & 0.55 & 0.55 & 0.06 & 0.09 & 0.09 \\
$112 \times 112(4)$ & 0.51 & 0.53 & 0.56 & 0.08 & 0.07 & 0.06 \\
$128 \times 128(3)$ & 0.48 & 0.55 & 0.57 & 0.06 & 0.07 & 0.06 \\
\hline \hline
\end{tabular}

above which the flow lines tend to infinity, corresponding to a fully localized state. Below $W_{r}$, the flow lines which start at finite disorder strength tend to a finite disorder strength of $W_{a} . W_{r}\left(W_{a}\right)$ is a repulsive (attractive) fixed point. We have done a number of calculations and this qualitative behavior is maintained; however, we found variation in the values of $W_{r}$ and $W_{a}$. Figure $3(\mathrm{~b})$ shows the size scaling exponent in 2D. In these calculations, system sizes up to $L=18$ ( $L$ is the linear dimension) were used and 100 replicas were averaged. Note that until $W \approx 1$, the size scaling exponent is approximately two; above that value it decreases. The flow lines in Fig. 2(b) are consistent with the behavior of the scaling exponent.

Similar behavior is found in 3D [see Figs. 2(c) and 3(c)], with a repulsive fixed point $W_{r}$ and an attractive fixed point $W_{a}$ and $W_{r}>W_{a}$. The flow lines shown in Fig. 2(c) are for an $8 \times 8 \times 8$ system with 100 replicas averaged.

In the G4 scaling theory, the system size itself appears as a relevant variable [Eq. (3)]. For this reason, we further investigate the system size dependence of the attractive and repulsive fixed points. Our results are shown in Tables I and II, and Fig. 4. To keep the CPU time manageable, we reduced the number of disorder configurations averaged, proportionately to system size. We made three calculations for each data point, and the raw data are shown in Tables I and II. A plot is shown in Fig. 4, with the raw results and their average.

In 3D, $W_{a}$ tends to zero and $W_{r}$ approaches a finite value with large system size. The function fit in Fig. 4(b) is $f(L)=a \exp (b L)+c$, where $a=31.8259, b=-0.489526$, and $c=4.75641$. A metal-insulator transition takes place at finite $W_{r}$, in agreement with the G4 [1]. In 2D, due to finite size limitations, a definite conclusion is difficult to reach. Several

TABLE II. The repulsive $\left(W_{r}\right)$ and attractive $\left(W_{a}\right)$ fixed points for three sample calculations as a function of system size for a threedimensional disordered system. The number of replicas is indicated in parentheses.

\begin{tabular}{lcccrrr}
\hline \hline Size (No. replicas) & $W_{r}(1)$ & $W_{r}(2)$ & $W_{r}(3)$ & $W_{a}(1)$ & $W_{a}(2)$ & $W_{a}(3)$ \\
\hline $8 \times 8 \times 8(100)$ & 5.49 & 5.46 & 5.23 & 0.42 & 0.40 & 0.49 \\
$12 \times 12 \times 12(25)$ & 4.96 & 4.87 & 4.73 & 0.30 & 0.28 & 0.25 \\
$16 \times 16 \times 16(10)$ & 4.60 & 4.96 & 4.68 & 0.08 & $<0.01$ & 0.23 \\
$20 \times 20 \times 20(5)$ & 5.05 & 4.70 & 4.58 & $<0.01$ & $<0.01$ & 0.05 \\
$24 \times 24 \times 24(3)$ & 4.62 & 4.97 & 4.66 & $<0.01$ & $<0.01$ & $<0.01$ \\
\hline \hline
\end{tabular}



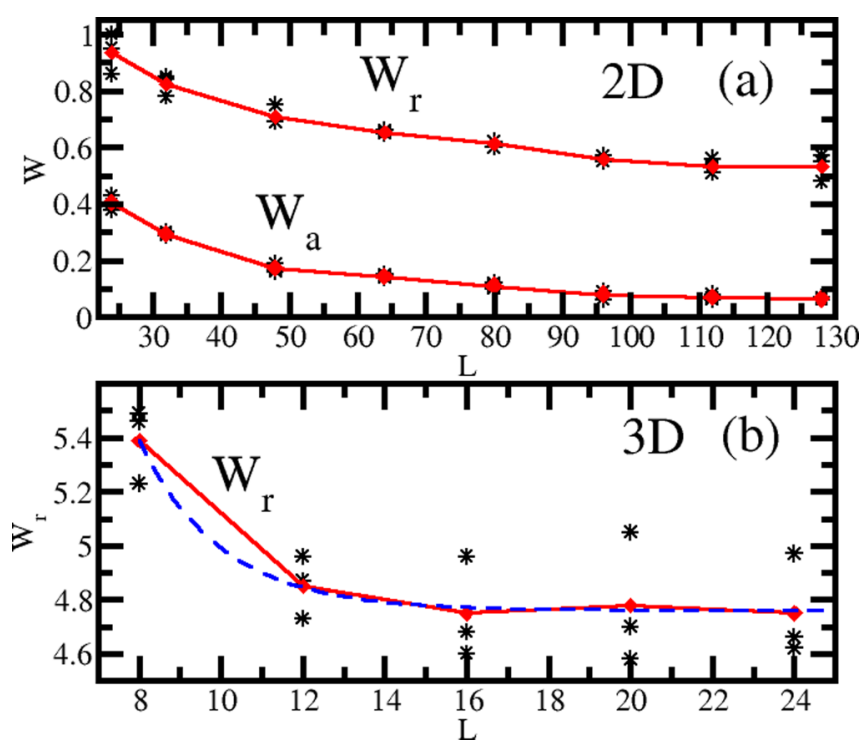

FIG. 4. The behavior of fixed points as a function of system size in $2 \mathrm{D}$ and $3 \mathrm{D}$. In $2 \mathrm{D}$, both the repulsive and the attractive fixed points are shown. The asterisks correspond to the raw data (three calculations) also shown in Tables I and II. The red diamonds show the average of the three calculations for each system size. The blue dashed curve for 3D shows a fit function (see the text for a detailed discussion).

scenarios are possible, depending on what happens to $W_{r}$ and $W_{a}$ as $L$ becomes large. Our results Fig. 4(a) show a sizable decrease for both $W_{r}$ and $W_{a}$, i.e., $W_{r} \approx 1$ for the smallest size and $W_{r} \approx 0.53$ for the largest one. Also, while $W_{a}$ does decrease, we cannot say that it reaches zero (as happens in 3D). There are then several possible scenarios. If $W_{a}$ and $W_{r}$ both go to zero, then extended states are absent, concurring with the G4. If only $W_{a}$ goes to zero, that would correspond to a metal-insulator transition, which would appear to be in accordance with transfer matrix and Lyapunov exponent based calculations $[14,16]$. A third possibility is that $W_{a}$ and $W_{r}$ both tend to finite values in the thermodynamic limit such that $W_{r}>W_{a}$. This would mean that there is a transition, but the small disorder states would be localized, rather than extended, even though they would scale with system size as $L$ [substitute a finite size dependent $Z_{1}$ in Eq. (10)]. We note that localized states of a qualitatively different nature, exhibiting power-law decay rather than exponential, have also been suggested [51] in 2D.
We also calculated $\gamma$ using different sets of systems, with the results shown as insets in Figs. 2(b) and 2(c). In 2D, we used systems with linear extension $L=12,16,18(L=$ $72,80,96)$ for the data points designated "small" ("large"). For the former, $\gamma$ remains two up to $W \approx 1.0$, while for the latter, the decrease from two starts earlier at $W \approx 0.6$. In $3 \mathrm{D}$, increasing the system size does not lead to a similar decrease. [Here the linear extensions were $L=6,8,10(L=20,22,24)$ for "small" ("large").] Although these results are also limited by system size limitations, the suggestion is that when the system size is increased, the curve in Fig. 3(b) eventually becomes like Fig. 3(a); in other words, no sign of extended states remains and our results likely concur with G4.

\section{CONCLUSIONS}

Wegner [52] is credited [2] with introducing concepts from statistical mechanics into the study of disordered systems. In this work, we applied statistical mechanical ideas using the characteristic function of the modern theory of polarization as a starting point. In particular, we derived a scaling relation according to the steps followed by Widom to relate critical exponents, and we applied a renormalization procedure to the problem of disorder. In $1 \mathrm{D}$ and $3 \mathrm{D}$, our method is in full agreement with the common wisdom [1] on Anderson localized systems; however, in 2D, we encountered system size limitations.

We note that the case of two dimensions has always been the most difficult one, both experimentally [10-13] and theoretically [14-16,51,53]. Although it is considered common knowledge that there are no extended states in two dimensions, the original work of Abrahams et al. [1] states that in spite of the absence of extended states, due to the crossover between exponential and logarithmic behavior, experiments may still detect a mobility edge.

\section{ACKNOWLEDGMENTS}

This research was supported by the Ministry of Innovation and Technology and the National Research, Development and Innovation Office (NKFIH), within the Quantum Information National Laboratory of Hungary and the Quantum Technology National Excellence Program (Project No. 20171.2.1-NKP-2017-00001). B.H. would like to thank Jan Wehr for a very enlightening discussion.
[1] E. Abrahams, P. W. Anderson, D. C. Licciardello, and T. V. Ramakrishnan, Phys. Rev. Lett. 42, 673 (1979).

[2] P. A. Lee and T. V. Ramakrishnan, Rev. Mod. Phys. 57, 287 (1985).

[3] F. Evers and A. D. Mirlin, Rev. Mod. Phys. 80, 1355 (2008).

[4] A. Langedijk, B. van Tieggelen, and D. S. Wiersma, Phys. Today 62(8), 24 (2009).

[5] J. Billy, V. Josse, Z. Zuo, A. Bernard, B. Hambrecht, P. Lugan, D. Clément, L. Sanchez-Palencia, P. Bouyer, and A. Aspect, Nature (London) 453, 891 (2008).
[6] G. Roati, C. D’Errico, L. Fallani, M. Fattori, C. Fort, M. Zaccanti, G. Modugno, M. Modugno, and M. Inguscio, Nature (London) 453, 895 (2008).

[7] S. S. Kondov, W. R. McGehee, J. J. Zibel, and B. DeMarco, Science 334, 66 (2011).

[8] F. Jendrzejewski, A. Bernard, K. Müller, P. Cheinet, V. Josse, M. Piraud, L. Pezzé, L. Sanchez-Palencia, A. Aspect, and P. Bouyer, Nat. Phys. 8, 398 (2011).

[9] G. Semeghini, M. Landini, P. Castilho, S. Roy, G. Spagnolli, A. Trenkwalder, M. Fattori, M. Inguscio, and G. Modugno, Nat. Phys. 11, 554 (2015). 
[10] D. H. White, T. A. Haase, D. J. Brown, M. D. Hoogerland, M. S. Najafabadi, J. L. Helm, C. Gies, D. Schumayer, and D. A. W. Hutchison, Nat. Commun. 11, 4942 (2020).

[11] M. Robert-de-Saint-Vincent, J.-P. Brantut, B. Allard, T. Plisson, L. Pezzé, L. Sanchez-Palencia, A. Aspect, T. Bourdel, and P. Bouyer, Phys. Rev. Lett. 104, 220602 (2010).

[12] F. Jendrzejewski, K. Müller, J. Richard, A. Date, T. Plisson, P. Bouyer, A. Aspect, and V. Josse, Phys. Rev. Lett. 109, 195302 (2012).

[13] K. Müller, J. Richard, V. V. Volchkov, V. Denechaud, P. Bouyer, A. Aspect, and V. Josse, Phys. Rev. Lett. 114, 205301 (2015).

[14] V. N. Kuzovkov, W. von Niessen, V. Kashcheyevs, and O. Hein, J. Phys.: Cond. Mater. 14, 13777 (2002).

[15] P. Markoš, L. Schweitzer, and M. Weyrauch, J. Phys.: Cond. Mater. 16, 1679 (2004).

[16] I. M. Suslov, J. Expt. Theor. Phys. 101, 661 (2005).

[17] R. D. King-Smith and D. Vanderbilt, Phys. Rev. B 47, R1651(R) (1993).

[18] R. Resta, Rev. Mod. Phys. 66, 899 (1994).

[19] R. Resta, J. Phys.: Cond. Mat. 12, R107(R) (2000).

[20] R. Resta, Phys. Rev. Lett. 80, 1800 (1998).

[21] R. Resta and S. Sorella, Phys. Rev. Lett. 82, 370 (1999).

[22] G. L. Bendazzoli, S. Evangelisti, A. Monari, and R. Resta, J. Chem. Phys. 133, 064703 (2010).

[23] V. Kerala Varma and S. Pilati, Phys. Rev. B 92, 134207 (2015).

[24] T. Olsen, R. Resta, and I. Souza, Phys. Rev. B 95, 045109 (2017).

[25] L. E. Reichl, A Modern Course in Statistical Mechanics, 4th ed. (Wiley-VCH, Weinheim, 2016).

[26] L. P. Kadanoff, Physics 2, 263 (1966).

[27] K. G. Wilson, Phys. Rev. B 4, 3174 (1971).

[28] R. Kobayashi, Y. O. Nakagawa, Y. Fukusumi, and M. Oshikawa, Phys. Rev. B 97, 165133 (2018).

[29] E. Domany and S. Sarker, Phys. Rev. B 20, 4726 (1979).

[30] S. Sarker and E. Domany, Phys. Rev. B 23, 6018 (1981).

[31] B. Shapiro, Phys. Rev. Lett. 48, 823 (1982).
[32] M. S. Foster, S. Ryu, and A. W. W. Ludwig, Phys. Rev. B 80, 075101 (2009).

[33] M. Nakamura and J. Voit, Phys. Rev. B 65, 153110 (2002).

[34] N. Marzari, A. A. Mostofi, J. R. Yates, I. Souza, and D. Vanderbilt, Rev. Mod. Phys. 84, 1419 (2012).

[35] M. Yahyavi and B. Hetényi, Phys. Rev. A 95, 062104 (2017).

[36] S. C. Furuya and M. Nakamura, Phys. Rev. B 99, 144426 (2019).

[37] S. Patankar, L. Wu, B. Lu, M. Rai, J. D. Tran, T. Morimoto, D. E. Parker, A. G. Grushin, N. L. Nair, J. G. Analytis, J. E. Moore, J. Orenstein, and D. H. Torchinsky, Phys. Rev. B 98, 165113 (2018).

[38] B. Hetényi and B. Dóra, Phys. Rev. B 99, 085126 (2019).

[39] B. A. Bernevig and T. L. Hughes, Topological Insulators and Superconductors (Princeton University Press, Princeton, NJ, 2013).

[40] L. Fu and C. L. Kane, Phys. Rev. B 74, 195312 (2006).

[41] J. T. Edwards and D. J. Thouless, J. Phys. C: Solid State Phys. 5, 807 (1972).

[42] W. Kohn, Phys. Rev. 133, A171 (1964).

[43] M. V. Berry, Proc. R. Soc. London A 392, 45 (1984).

[44] J. Zak, Phys. Rev. Lett. 62, 2747 (1989).

[45] A. Selloni, P. Carnevali, R. Car, and M. Parrinello, Phys. Rev. Lett. 59, 823 (1987).

[46] E. S. Fois, A. Selloni, M. Parrinello, and R. Car, J. Chem. Phys. 92, 3268 (1988).

[47] F. Ancilotto and F. Toigo, Phys. Rev. A 45, 4015 (1992).

[48] W. P. Su, J. R. Schrieffer, and A. J. Heeger, Phys. Rev. Lett. 42, 1698 (1979).

[49] M. J. Rice and E. J. Mele, Phys. Rev. Lett. 49, 1455 (1982).

[50] Th. Niemeijer and J. M. J. van Leeuwen, Phys. Rev. Lett. 31, 1411 (1973).

[51] N. F. Mott and M. Kaveh, Adv. Phys. 34, 329 (1985).

[52] F. J. Wegner, Z. Phys. B 25, 327 (1976).

[53] V. Srivastava, Phys. Rev. B 41, 5667 (1990). 